\title{
Corpus
}

\section{Le discours de Tony Blair (1997-2004)}

\section{Edward Arnold}

\section{(2) OpenEdition}

1 Journals

\section{Édition électronique}

URL : http://journals.openedition.org/corpus/340

DOI : $10.4000 /$ corpus.340

ISSN : $1765-3126$

\section{Éditeur}

Bases; corpus et langage - UMR 6039

\section{Édition imprimée}

Date de publication : 1 décembre 2005

ISSN : 1638-9808

Référence électronique

Edward Arnold, «Le discours de Tony Blair (1997-2004) », Corpus [En ligne], 4 | 2005, mis en ligne le 01 septembre 2006, consulté le 07 septembre 2020. URL : http://journals.openedition.org/corpus/340 ; DOI : https://doi.org/10.4000/corpus.340

Ce document a été généré automatiquement le 7 septembre 2020

(c) Tous droits réservés 


\title{
Le discours de Tony Blair (1997-2004)
}

\author{
Edward Arnold
}

Introduction

1 On a souvent dit que «Gouverner c'est parler ». Même si cette formule est exagérée, il est certain que la science politique contemporaine ne peut se désintéresser des discours tenus par les acteurs. Elle ne peut donc faire l'économie d'une réflexion concernant le recueil raisonné de ces discours - la constitution de corpus - et concernant l'exploitation de ces collections de textes à l'aide de sciences comme la linguistique, la statistique appliquée ou la gestion des bases de données ${ }^{1}$.

2 Nous allons illustrer cette discussion à l'aide d'un exemple : le corpus des interventions du Premier ministre anglais T. Blair depuis son arrivée au pouvoir en 1997. Durant ces huit ans, la nature des rapports entre les gouvernants et les gouvernés a été bouleversée, notamment du fait de l'importance prise par le «spin » - ce terme désigne l'activité des conseillers, des spécialistes en communication, en relations publiques, qui sont chargés de l'image des partis et des leaders politiques -, importance que certains jugent excessive. Par exemple, en décembre 2004, Lord Butler, ancien Secrétaire du Conseil des ministres et ancien Secrétaire général de la Fonction publique a publiquement estimé que T. Blair donne trop de poids à ces conseillers, dépourvus de mandat démocratique et que, en revanche, il n'a pas toujours tenu compte des avis des ministres et des hauts fonctionnaires dans la conception et dans la rédaction des nombreuses lois dont le New Labour a été l'instigateur depuis 1997 [Butler 2004]. De très nombreux ouvrages ont été consacrés au personnage, à son mode de gouvernement (par exemple : [Abse 2003] ; [Beckett \& Hencke 2003] ; [Hamilton 2000] ; [Rawnsley 2004]; [Riddell 2004]; [Seldon 2004]), à son discours ([Fairclough 2000]; [Richards 2004]), avec, ces dernières années, une insistance particulière sur les guerres menées par les Travaillistes ([Kampfner 2003] ; [Short 2004]).

3 Le rassemblement des interventions de toutes natures, effectuées par T. Blair depuis 1997, a été la première étape d'un projet consistant à constituer un large corpus du discours politique anglais contemporain qui devrait également s'étendre au discours 
politique irlandais (en anglais) et aboutir à une base de données textuelles aisément consultable avec l'espoir de pouvoir placer ce corpus en ligne suivant l'exemple de PoliText, la base de données conçue à l'Université de Nice au laboratoire Bases, Corpus et Langage (CNRS-ILF), sur les discours politiques français (http://www.unice.fr/ILFCNRS/ politext/). Cet article tire un bilan de la première étape qui a permis d'inventorier les problèmes, de dessiner les caractéristiques de la future base et d'entrevoir ses intérêts.

1. Le corpus

4 Le corpus actuel comporte 237 textes représentant autant d'interventions de T. Blair depuis qu'il est devenu Premier ministre en 1997. Ces textes ont été récupérés, depuis un an, sur le site officiel du 10 Downing Street. Chacun des documents "originaux " (en fait le fichier électronique) est conservé dans les « archives du corpus ». En effet, il s'agit de documents en ligne, donc périssables. A tout moment, le fichier électronique peut disparaître. Cela est particulièrement vrai pour un site comme celui du 10 Downing Street qui est organisé de manière thématique et n'a pas vocation à l'exhaustivité. Lors des mises à jour, il arrive même que certains textes soient retirés, probablement parce qu'ils sont jugés trop anciens ou obsolètes. C'est la raison pour laquelle les deux dernières années occupent pratiquement la moitié du corpus actuel. Il devra donc certainement être complété par la saisie d'archives papier. Enfin, il est indispensable de pouvoir retourner, à tout moment, à la source, car les opérations de préparation et de balisage, qui permettent son intégration dans le corpus proprement dit, ne sont pas bénignes.

\subsection{Préparation du texte}

5 Avant son intégration dans le corpus, un triple travail préliminaire, fastidieux mais indispensable, est effectué sur chaque texte.

6 En premier lieu, l'orthographe est revue. Certes, il y a peu de fautes de frappe et d'orthographe dans les textes mis en ligne par le 10 Downing street, mais ce contrôle doit, malgré tout, être fait avec soin. En effet, une base électronique de textes destinée à des usages scientifiques ne peut pas contenir de fautes, car ces fautes génèrent des « fantômes de mots » qui faussent les statistiques.

7 Deuxièmement, les abréviations et les graphies non conventionnelles sont standardisées. En effet, avant de constituer un corpus électronique, il est indispensable de répondre, une fois pour toutes à cette question : que faire avec les très nombreux « $1^{\text {st }} », 《 2^{\text {nd }} », ~ « 3^{\text {rd }} »$, «bn», « $\mathrm{Mr} »$, etc. Les conserver tels quels? Cela éviterait un travail fastidieux qui comporte le double risque d'oublier certaines de ces graphies insolites et d'introduire des fautes, risque inhérent à toute intervention manuelle sur un texte. Mais alors : il y aura (au moins) deux mots différents pour " first ", « second », «third », «billion » ou « $\mathrm{Mr}$ » (avec ou sans point). De manière générale, les numéraux, les cardinaux et les unités de mesure posent également de redoutables problèmes. Etant donné l'usage abondant des chiffres, sigles et abréviations dans le discours politique contemporain, quelle sera la crédibilité de statistiques réalisées sur ces formes graphiques enregistrées sans aucun travail de remise en ordre?

Troisièmement, l'orthographe des noms propres est standardisée (surtout les transcriptions de l'arabe, de l'hindi ou du chinois...) afin que la même personne n'apparaissent pas sous deux ou trois noms faussement différents.

9 Ne pas intervenir à ce triple niveau rendrait la future base difficile à utiliser et les index, outils indispensables à la plupart des recherches, seraient remplis de 
«fantômes » de mots... Au moins, ce type de corpus présente un avantage considérable par rapport aux textes littéraires : on ne "corrige " pas le Premier ministre mais la secrétaire qui a transcrit ses propos. Ajoutons que, lorsque la base sera en activité, un document annexe résumera les principales interventions effectuées et les conventions suivies lors de ces interventions. C'est aussi pourquoi le document original sera joint à la base, cela permettra à l'utilisateur de contrôler le bien-fondé des interventions. Au passage: cette règle de transparence semble bien peu souvent respectée par les réalisateurs de corpus.

Enfin, pour mémoire, évoquons le problème de la norme d'indexation et de dépouillement du corpus. S'il est évident que « The » et « the » doivent être rassemblés sous la même entrée, jusqu'où doit-on aller dans cette voie? La lemmatisation fait l'objet d'un vif débat, notamment au sein de la communauté des linguistes de corpus (Knowles 2004). Dans la phase préparatoire actuelle, signalons simplement que nous avons utilisé la norme la plus répandue - Oxford Concordance [Hockey \& Martin : 1988] - qui opère au niveau des formes graphiques.

11 Une fois franchie cette première étape, le texte subit un encodage qui permettra son intégration au sein de la base.

1.2. Encodage

Il s'agit d'un encodage minimal à deux niveaux :

Premièrement, les passages attribuables au Premier ministre sont isolés de tout le reste. En effet, dans ces textes, beaucoup de choses ne sont pas, à proprement parler, «du Tony Blair » : le titre et le résumé, voire des intertitres, que les services du Premier ministre ont parfois ajoutés au texte, les questions des journalistes ou des téléspectateurs lors des entretiens, les réponses des autres chefs d'Etat ou de gouvernement qui participaient aux «conférences de presse conjointes » avec T. Blair, et aussi certains ajouts du sténographe : «(rire)», «(inaudible)», etc. Il est essentiel que ces parties de texte ne soient pas présentées à l'utilisateur comme ayant été prononcées ou écrites par le Premier ministre, mais il est aussi important que cet utilisateur puisse y accéder pour la bonne compréhension des passages qui lui sont retournés. En quelque sorte, le problème est identique à celui que posent les pièces de théâtre : une partie du texte les indications scéniques, les noms des personnages qui parlent, etc. - est un «cotexte ", ou mieux un "para-texte ", qui doit être conservé tout en étant nettement distingué du texte proprement dit. Pour cela, nous avons suivi l'usage minimal qui consiste à placer ce « para-texte » entre < > en ajoutant, juste après le crochet ouvrant, des indications standardisées du type " titre ", " entête ", " question ", " interruption ». Dans l'étape actuelle, cela permet de neutraliser le "para-texte ", tout en préparant l'étape ultérieure : une fois ces références définies une fois pour toutes, le début et la fin de ces passages spécifiques pourront être transformés aisément en véritables balises hyper-textuelles.

Deuxièmement, quelques attributs sont affectés à chaque texte. En plus du locuteur puisque la future base est destinée à accueillir beaucoup d'autres hommes politiques et de la source, il s'agit de : la date, le lieu et la nature du texte. Toutes ces indications devant être fournies à l'usager qui extrait un texte de la base.

Lorsque ces futurs corpus seront intégrés dans une véritable base de données, cet encodage permettra de naviguer dans la base, comme on navigue sur internet, en rendant possible une série de requêtes: la recherche d'un mot, d'une combinaison de mots dans toute la base mais aussi dans une partie seulement de la base. Par exemple, 
tous les textes émis par tel homme politique, quelle que soit leur nature, entre telle et telle date. Ou bien, on pourra se demander quel est le vocabulaire caractéristique des « discours du Trône »; etc.

Sans doute, ces considérations sembleront-elles banales pour ceux qui ont déjà participé à la constitution d'un corpus, mais elles surprennent toujours le profane qui s'imagine qu'il suffit d'« enfourner» les textes dans un ordinateur pour constituer un corpus, voire une base de données !

1.3. Etat actuel du corpus « Tony Blair »

Au stade actuel, le corpus « Tony Blair » comporte 237 textes, soit 450674 « tokens » et 12657 «types» (sous réserve d'ultimes corrections). A ce propos, il ne semble pas y avoir, en français, d'expressions capables de rendre l'idée simple selon laquelle le texte est constitué de $\mathrm{N}$ « cases » successives occupées par autant de « jetons » qui sont de $\mathrm{V}$ "types» différents. En statistique textuelle, spécialité typiquement française, on trouve bien: "occurrence» vs. "forme (graphique)». Malheureusement, « occurrence» est surtout employé comme synonyme de "fréquence absolue » («le nombre d'occurrences d'un mot»). Quand à " forme ", il sert aussi bien à désigner un "emplacement" dans le texte, qu'une graphie particulière, une flexion, voire une « entrée de dictionnaire " (" headword », « vocable »)... Nous sommes donc condamnés à suivre l'usage, incommode mais dominant, qui parle de "mot» (pour traduire " token ») et de " mot différent » (pour traduire « type »).

Parmi les textes du corpus, la conférence de presse du 22 avril 2004 est le plus long (8 706 mots) ; le plus court (153 mots) n'est pas le moins important puisqu'il s'agit de la déclaration conjointe avec le Premier ministre d'Irlande qui ouvre les négociations sur la paix en Irlande du nord (26 février 1998). La moyenne est inférieure à 2000 mots (médiane: 1500 mots): dans l'ensemble, ce sont donc des textes courts. Dans le discours politique anglais, il n'existe rien de semblable - quant à l'ampleur des vues et à la longueur de l'exercice - aux déclarations des Premiers ministres français ou aux conférences de presse élyséennes.

19 A l'aide de ce corpus provisoire, nous allons tenter d'illustrer quelques-uns des intérêts multiples de cette base (sur les intérêts de la linguistique de corpus, voir [Habert, Nazarenko \& Salem 1997], et le premier numéro de Corpus). En premier lieu, il n'est pas inutile de rappeler qu'il s'agit de fournir aux spécialistes en sciences humaines et sociales, les moyens d'accéder facilement à un ou plusieurs discours ou à des renseignements plus spécifiques comme: quelles sont les attestations d'un mot ou d'une famille de mots dans tout ou partie de la base ? Au-delà de cet aspect évident, on doit pouvoir tirer d'une base électronique de textes, des indications précises concernant le vocabulaire du ou des auteur(s) : index des mots différents classés par ordre alphabétique (ou par fréquences), concordance de certains vocables dont on recherche le sens spécifique, vocabulaire caractéristique d'un texte ou d'une certaine catégorie de textes, etc. Pourtant, aujourd'hui, combien de « corpus» sont réellement capables de répondre à ces attentes simples? Si le travail préalable de correction et de standardisation des graphies n'a pas été effectué, comment être certain d'avoir toutes les attestations du mot recherché ? etc. Ce genre d'interrogations suffit amplement à justifier le travail préalable qui vient d'être évoqué. Mais il faut aller plus loin. Le principal intérêt de ces corpus réside dans les voies nouvelles qu'ils ouvrent, dont la plupart sont encore à défricher mais qui promettent d'être fort intéressantes pour les linguistes et, aussi, pour tous les chercheurs en sciences humaines et sociales, 
spécialement les politologues. Nous allons illustrer cette dernière idée à l'aide de deux exemples: le repérage des principales ruptures thématiques et stylistiques dans les 8 années de présence au pouvoir de $\mathrm{T}$. Blair et la richesse de son vocabulaire.

2. Les ruptures thématiques et stylistiques dans le discours de T. Blair

Peut-on distinguer plusieurs périodes dans le discours de T. Blair entre 1997 et 2004 ? Cette question se pose à chaque fois que l'on se trouve devant un corpus dans lequel on suppose qu'il existe une dimension chronologique, ce qui est généralement le cas pour les corpus politiques, que l'on imagine volontiers «traversés par l'Histoire » (sur cette discussion, voir [Prost 1988] et une synthèse dans [Guilhaumou 2002]).

21 Pour répondre à cette question, la plupart des analyses font appel à des critères externes au discours, comme les "évènements marquants »: on classe les discours suivant qu'ils ont été tenus avant ou après l'événement que l'on suppose décisif et l'on demande aux données textuelles de valider (ou d'invalider) cette hypothèse; d'autres se contentent de découper le corpus en "années politiques", en utilisant les dates anniversaires de l'élection ou de l'arrivée au pouvoir de l'auteur, voire en "années civiles » (par exemple [Mayaffre 2000 et 2004]). Ne serait-il pas préférable d'opérer ces coupures en utilisant des critères internes aux textes et sans aucun a priori ? De ce point de vue, deux mesures fournissent des éléments intéressants pour l'historien: l'accroissement du vocabulaire et la diversité du vocabulaire.

\subsection{L'accroissement du vocabulaire}

Dans tout texte en langue naturelle, l'afflux de mots nouveaux est d'abord extrêmement fort puis il décroît rapidement, tout en demeurant positif, même dans les textes extrêmement longs. Depuis les travaux de [G. Herdan 1960], cette question est connue sous le nom de "type-token ratio ". Les modèles proposés postulent généralement que le texte est issu d'un vocabulaire unique qui s'épuise progressivement au fur et à mesure que le texte s'allonge (par exemple [Muller 1977]). [Hubert \& Labbé 1988] ont proposé un modèle à "vocabulaires multiples»: un «vocabulaire général» comportant les mots qui sont employés quel que soit le contexte (la croissance de ce vocabulaire est décrite par la loi hypergéométrique) et une série de "vocabulaires spécialisés » comportant les mots qui sont utilisés pour traiter un thème particulier (l'économie, la monnaie, la diplomatie...). La croissance de ce second ensemble suit une tendance linéaire. Le calcul est relativement simple et les résultats «collent » bien aux données observées comme on peut le constater dans le graphique 1 ci-dessous.

23 La courbe d'ajustement passe exactement au milieu du nuage de points, ce qui rend les deux traits difficiles à différencier, sauf à trois reprises. Un peu avant 100000 mots et entre 300000 et 350000 mots, les valeurs observées passent visiblement au-dessus des valeurs théoriques; vers 250000 mots, ces valeurs sont au-dessous de la courbe " idéale ». Ces « accidents » semblent ténus : rien ne permet d'affirmer que les écarts sont significatifs. Pour surmonter cette difficulté, [Labbé, Labbé \& Hubert 2004] préconisent l'emploi d'une méthode issue de l'analyse des séries statistiques présentant une composante « cyclique » (statistiques économiques, clima-tiques, etc). 
Graphique 1. Courbe observée et courbe ajustée de l'accroissement du vocabulaire dans l'ensemble du corpus « Tony Blair»

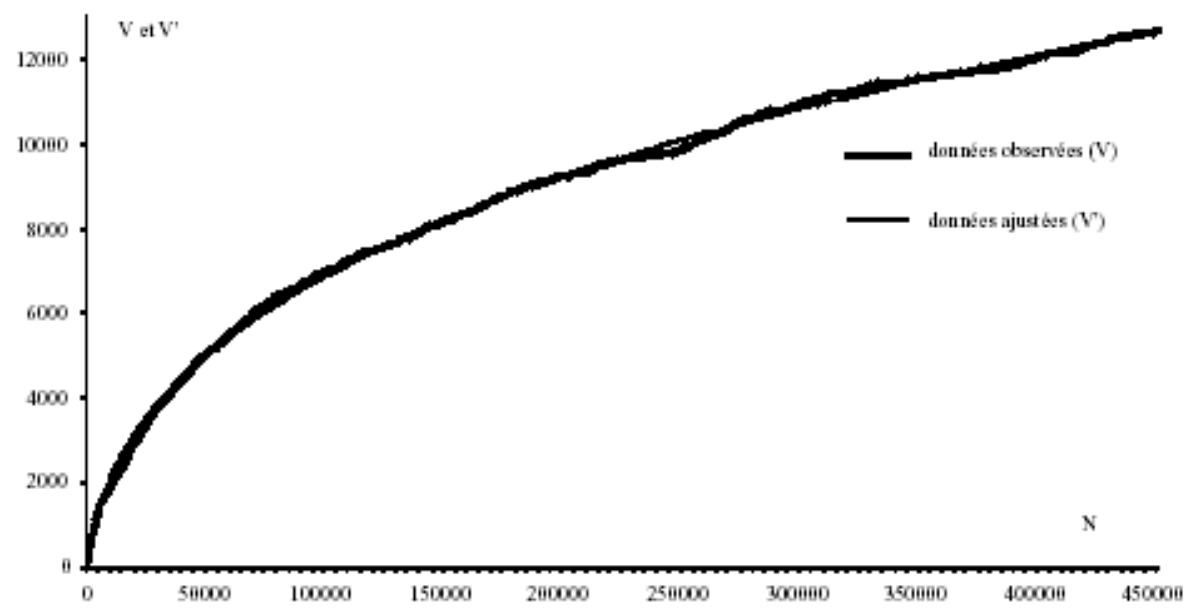

En premier lieu, les données observées (le trait gras) sont «centrées » sur la tendance (le trait maigre) qui devient l'axe horizontal du graphique 2 (chaque observation est soustraite de la valeur théorique correspondante). Puis ces données "centrées » sont « réduites » ou « normées » (en les divisant par l'écart type). Cette dernière opération permet de savoir si les observations s'écartent ou non de ce que donneraient de simples variations aléatoires autour de la tendance : au-dessus de 2 et en dessous de -2 (sur l'axe des $\mathrm{x}$ ), on peut affirmer que les variations ne sont pas dûes au hasard (avec moins de 5 chances sur 100 de se tromper). Si T. Blair avait toujours suivi le même rythme moyen, tous les points de la courbe seraient compris dans cet intervalle.

Le graphique 2 indique des mouvements relatifs. Une portion de courbe ascendante signale qu'à cet endroit se produit un afflux "anormal» de mots nouveaux, ce qui laisse supposer un renouvellement thématique. A l'inverse, une baisse est le signe que «trop peu » de mots nouveaux apparaissent: l'orateur répète des choses déjà dites auparavant, il épuise des thèmes anciens. 


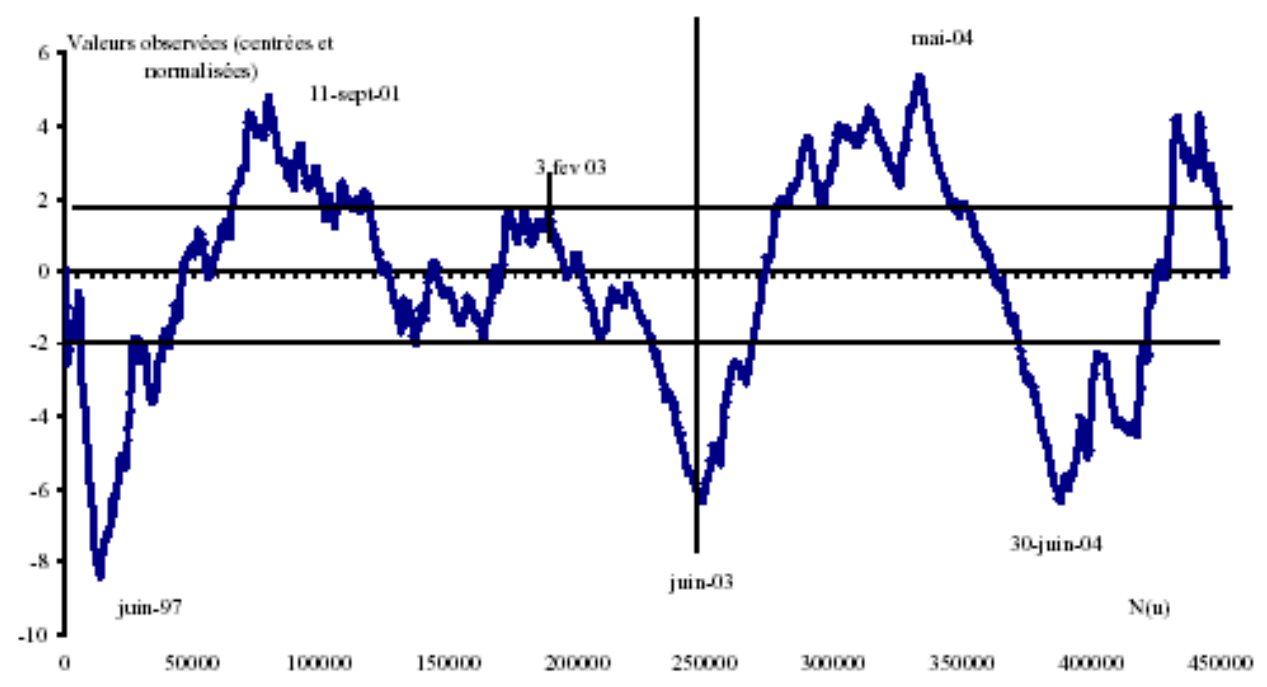

La courbe est «contrainte» à ses deux extrémités (elle commence et se termine nécessairement à l'origine). De plus, près des extrémités, l'écart type est très petit. Ces deux éléments expliquent que, si lors des premiers mois de pouvoir (mai-juin 1997), comme pendant les derniers jours de 2004, le discours de T. Blair connaît un " passage à vide", celui-ci n'a peut-être pas tout à fait l'ampleur que suggère le graphique. Cependant, il est intéressant de noter que la faiblesse des premières semaines correspond à trois interventions, spécialement un long entretien à propos de la « loi et l'ordre ", puis une séance de questions-réponses devant la Chambre à propos de l'Europe. A chaque fois que, au cours des 7 ans qui suivent, T. Blair a abordé particulièrement ces deux problèmes, on constate une « encoche » dans la courbe, plus ou moins significative en fonction de la longueur de ces interventions. Autrement dit, sur ces deux questions, le Premier ministre a eu tendance à s'en tenir à des positions «standards » et il a peu renouvelé son vocabulaire. Est-ce par désintérêt personnel, embarras politique, crainte de perdre une partie de ses électeurs ou de diviser inutilement son parti? D'autres analyses seront nécessaires pour répondre à ces questions.

Le tournant de juin 1997 correspond à un discours devant l'ONU suivi d'un entretien sur la réforme de l'éducation. Tout au long de ces huit ans, T. Blair a toujours semblé plus à l'aise lorsqu'il était question des relations internationales (autres que l'Europe et l'Irak!) - spécialement les relations du Royaume-Uni avec les Etats-Unis et le Commonwealth -, et sur certains sujets de politique intérieure comme la réforme des services publics, spécialement l'éducation.

Un autre «tournant» se situe clairement au lendemain du 11 septembre 2001: le Premier ministre se lance dans plusieurs tournées à travers le monde avec un seul thème pour ses discours et ses conférences de presse: la mobilisation contre le terrorisme et la préparation de la guerre en Afghanistan. La pente négative de la courbe à partir de cette date marque bien cette répétition - juste interrompue le temps d'un grand discours sur la "Public Service Reform " ( $1^{\text {er }}$ octobre 2001). De même, pendant tout le premier semestre 2003, c'est l'Irak qui tient le devant de la scène et l'on observe le même profil de courbe: peu de mots nouveaux apparaissent, le Premier ministre répète inlassablement les mêmes formules. Enfin, le phénomène se reproduit 
durant le printemps 2004, lorsque l'Irak revient au premier plan de l'actualité, notamment avec la polémique autour de l'existence des «armes de destruction massive » qui avaient justifié l'intervention américano-britannique.

D'après le graphique 2, le tournant majeur de ces 7 années semble se situer en juin 2003 avec le sommet du G8 à Evian, suivi de la conférence annuelle du Labour en septembre, deux événements qui ont été l'occasion d'aborder de nouveaux thèmes, et qui ont permis au Premier ministre de reprendre l'offensive, tout en reléguant l'Irak au second plan (du moins pendant six mois...).

Ainsi, chaque "accident » de la courbe peut être référé à un texte - voire un passage précis dans un texte - et à une situation politique particulière. Les principaux points d'inflexion de la courbe permettent également de découper les périodes sans avoir recours à des hypothèses préalables concernant l'histoire contemporaine. Cette analyse du vocabulaire peut être complétée par l'étude de la dimension stylistique.

\subsection{La diversité du vocabulaire}

Pour approcher la dimension stylistique, il est proposé d'utiliser un indice simple : la diversité du vocabulaire, c'est-à-dire le nombre de mots différents employés dans un court empan de mots (de quelques centaines de mots à un millier). Le graphique 3 cidessous décrit l'évolution de cette diversité au long des huit années de gouvernement Blair. La variable est mesurée sur un empan de mille mots, elle est centrée sur la moyenne et normée selon la même méthode que précédemment.

Graphique 3. Evolution de la diversité du vocabulaire dans les discours de T. Blair (1997-2004). Tranches de 1000 mots. Variable centrée et réduite

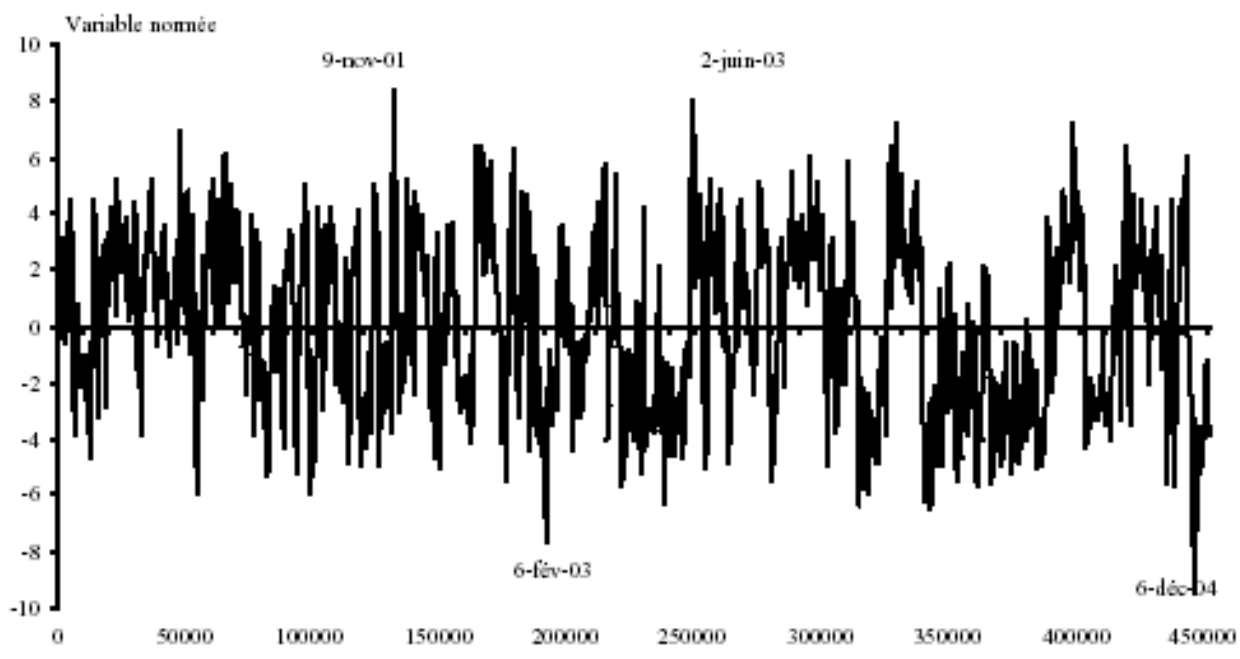

Le phénomène se révèle fortement instable et la majorité des valeurs sortent de l'intervalle de fluctuation «normal »- compris entre les ordonnées \pm 2 - par le haut ou par le bas. Ce profil n'est pas propre à T. Blair : dans les corpus français, on observe des choses semblables pour tous les locuteurs, du moins lorsque la performance est longue (voir les exemples donnés par [Labbé, Labbé \& Hubert 2004]).

On peut d'abord relever les points exceptionnels. Par exemple, la diversité maximale (à près de 9 écarts types au-dessus de la moyenne) est atteinte dans la tranche 131-132 000 mots, c'est-à-dire le 9 novembre 2001, lors d'un bref entretien à bâtons rompus avec la presse à la sortie d'une rencontre avec le président du Pakistan, Musharraf. La 
discussion portait sur les conséquences des attentats du 11 septembre 2001, la lutte contre le terrorisme, les relations de l'Occident avec l'Islam et la préparation de la guerre en Afghanistan (le soutien du Pakistan était indispensable). Durant près de deux mois, le Premier ministre s'était entièrement consacré à cette question; il pensait la posséder dans toute sa complexité et estimait, probablement, qu'une solution avait été trouvée. Il a réalisé lors de cet oral "spontané», une performance nettement supérieure à celle dont il a été capable dans tous ses autres discours, au cours de la période, y compris les écrits.

31 Le second indice le plus élevé est atteint à la fin de la conférence de presse qu'il donne à l'issue du sommet du G8 à Evian (2 juin 2003), conférence au cours de laquelle les principales puissances ont paru approuver l'intervention américano-britannique en Irak, ce qui semblait donner au Royaume-Uni une place de premier plan dans le nouvel ordre mondial.

L'indice le plus bas est atteint à la fin de la période étudiée, le 6 décembre 2004, lors d'une conférence de presse conjointe avec... le président Musharraf qui portait, apparemment, à peu près sur les mêmes grands thèmes que trois ans auparavant ! L'autre point bas (191000 mots) survient le 6 février 2003, à la veille de la guerre en Irak, lors d'une émission télévisée en direct au cours de laquelle T. Blair a été malmené par les animateurs et des spectateurs sceptiques envers les justifications qu'il présentait en faveur de cette guerre.

Ces quatre exemples auront suffi pour montrer combien l'indice de la diversité du vocabulaire est un puissant révélateur de la situation du locuteur, de sa relation intellectuelle avec les thèmes traités, et de ses choix de communication (on pourrait presque dire, à propos des deux derniers exemples cités, qu'il s'agissait d'un choix de "non-communication»). Il suffira donc de relever systématiquement tous les points "anormaux» (du point de vue statistique), les textes ou passages de textes correspondants - ainsi que le média utilisé - pour dresser un tableau éclairant des «hauts et des bas » de la communication du Premier ministre depuis son arrivée au pouvoir. Naturellement, ces mesures ponctuelles ne donnent pas d'indications concernant son "style" personnel... Pour ce faire, il faudrait disposer des mêmes mesures pour d'autres hommes politiques anglais contemporains.

En face d'un phénomène d'aspect « chaotique », comme celui que décrit le graphique 3 , la question se pose de savoir s'il existe un "ordre caché », ou du moins, s'il est possible d'isoler quelques épisodes relativement stables. Une méthode dite de «segmentation automatique " permet de répondre à cette question complexe. Elle a été mise au point en climatologie ([Hubert, Charbonnel \& Chaouche 1989]), où les données présentent souvent un profil semblable à celui du graphique 3. Elle a été adaptée aux données textuelles par [Péaquin 2003], ou [Labbé, Labbé \& Hubert 2002 et 2004]. Appliquée à la série décrite dans le graphique 3 , cette procédure aboutit à la mise en évidence de neuf périodes significatives (trait gras sur le graphique 4). 
Graphique 4. Segmentation automatique des interventions de T. Blair, classées par ordre chronologique, en fonction de la diversité du vocabulaire

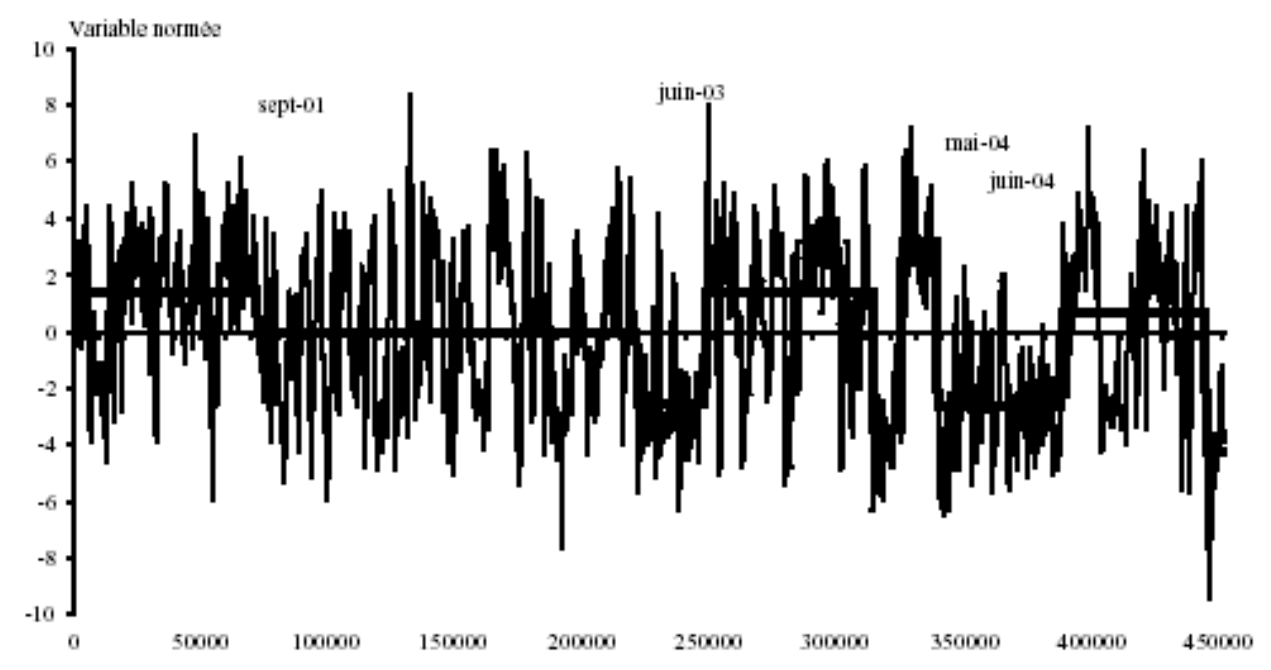

Il faut garder présent à l'esprit le fait que l'algorithme utilise un gros " grain » (1000 mots, ce qui donne tout de même 450 points sur le graphique) car il s'agit ici d'avoir une vue d'ensemble de l'ère T. Blair, tout en conservant une certaine lisibilité. La même technique peut être utilisée à un niveau plus fin (un texte long, une courte période). Pour la même raison, on impose à l'automate de constituer des blocs de taille supérieure à 10000 mots (afin que le calcul de la variance entre segments se fasse sur un nombre suffisant de valeurs). Ainsi le "passage à vide» de juin 1997, visible au début des graphiques 3 et 4 , n'a pas été isolé comme un segment significatif, car il est trop bref.

La superposition des graphiques 2 et 4 , montre que la "composante stylistique " épouse presque parfaitement l'évolution de la «composante lexicale ». Les 3 encoches nettement inférieures à la moyenne, qui signalent un affaiblissement significatif de la diversité de l'expression, correspondent exactement aux périodes "guerrières " qui ont été localisées en observant l'accroissement $d u$ vocabulaire. Les tournants significatifs sont localisés aux mêmes endroits. Les deux analyses conduisent en définitive à délimiter deux périodes principales (avant et après juin 2003), la seconde étant plus heurtée, comme si le Premier ministre ne parvenait pas à retrouver l'équilibre relatif des années antérieures à la guerre en Irak. Chacune de ces deux grandes périodes peut ensuite faire l'objet d'une segmentation plus détaillée, en utilisant un niveau fin.

Les coupures étant maintenant opérées avec certitude, on peut établir le vocabulaire de chacune des périodes et, à l'aide de la technique bien connue des spécificités du vocabulaire, on peut mettre au jour les thèmes dominants et les singularités (ce point ne peut être développé faute de place et fera l'objet d'une autre publication en cours de préparation).

3. Oral et écrit à travers la diversité et la spécialisation du vocabulaire

37 Les calculs présentés ci-dessus apportent également une solution intéressante à la vieille discussion autour de la richesse du vocabulaire d'un texte ou d'un auteur ([Wimmer \& Altmann 1999]). Cette richesse sera mesurée à l'aide de deux indicateurs complémentaires ([Hubert \& Labbé 1994]) : l'indice de diversité (qui vient d'être 
présenté) et l'indice de spécialisation, c'est-à-dire le poids relatif du vocabulaire spécialisé dans le total du vocabulaire. Cette dernière mesure indique la propension à spécialiser le discours en fonction du thème traité. Sur les corpus français, les indices inférieurs ou égaux à $10 \%$ caractériseraient une propension faible à spécialiser le propos ; entre 10 et 20 cette propension serait moyenne et au-dessus de $20 \%$ elle serait forte ([Labbé \& Monière 2003]).

Pour l'ensemble du corpus, T. Blair fait preuve d'une spécialisation quasi-nulle (taux à peine supérieur à $1 \%$ ) comme beaucoup d'hommes politiques contemporains (voir par exemple, à ce sujet, les chefs de gouvernement français, canadiens et québécois étudiés par Labbé et Monière). On peut supposer qu'il s'agit d'un fait stylistique (une certaine propension à se réfugier dans les généralités) mais, dans ce cas, cette même caractéristique devrait se retrouver dans chaque discours, conférence de presse, etc. Ce n'est manifestement pas le cas en ce qui concerne les discours du Trône (première colonne du tableau 1).

Tableau 1. Spécialisation et diversité du vocabulaire dans les discours du Trône des gouvernements de T. Blair

\begin{tabular}{|l|l|l|l|}
\hline & Spécialisation (\%) & Diversité (\%) & Taille \\
\hline \hline 1997 & 48 & 425 & 1437 \\
\hline \hline 1998 & 183 & 414 & 1602 \\
\hline \hline 1999 & 120 & 404 & 953 \\
\hline \hline 2000 & 40 & 392 & 953 \\
\hline \hline 2001 & 72 & 406 & 160 \\
\hline \hline 2002 & 93 & 407 & 197 \\
\hline \hline 2003 & 113 & 406 & 1437 \\
\hline \hline 2004 & 287 & 406 & 1208 \\
\hline \hline Total & 3 & & 10535 \\
\hline
\end{tabular}

(valeurs exprimées en « pour mille mots »)

La spécialisation interne à chaque discours du Trône est toujours significative. Elle est faible pour trois d'entre eux (dont le premier), moyenne pour les autres et même forte dans le dernier (sous réserve que l'échelle proposée pour le français soit applicable à l'anglais). Or ce même indice de la spécialisation, mesuré sur l'ensemble des discours du Trône mis bout à bout, apparaît quasi nul (dernière ligne du tableau 1). Ce paradoxe peut s'expliquer de la manière suivante. Tous les discours du Trône sont construits de la même manière et comportent d'ailleurs un certain nombre de formules rituelles, quasiment figées, placées à peu près toujours aux mêmes endroits. Une même structure se répète à espaces réguliers dans des discours de dimensions voisines. Cette répétition 
aboutit à un ensemble apparemment sans spécialisation. Autrement dit, si l'on se représente les indices de spécialisation du vocabulaire de ces différents textes, mis bout à bout, comme un nuage de points, les paramètres d'ajustement du nuage entier ne renseignent pas sur les qualités spécifiques des différentes parties de ce nuage. La spécialisation quasi nulle qui a été observée sur l'ensemble du corpus pourrait provenir d'un phénomène semblable. En effet, au long des années 1997-2004, T. Blair a rencontré une série de problèmes: l'Irlande du Nord, l'Europe, l'Irak et, au plan intérieur: la réforme de l'Etat providence, de l'éducation, des services publics; l'insécurité et la politique pénale. Sauf durant quelques courtes périodes - qui ont été dominées par une question majeure : les suites du 11 septembre, puis la guerre en Irak et ses conséquences -, ces questions sont revenues de manière régulière et, en gros, la réponse du Premier ministre a été peu différente.

On peut vérifier cette intuition en examinant les valeurs prises par cette même variable lors de ses principales prestations orales (tableau 2).

Tableau 2. Spécialisation et diversité du vocabulaire dans les conférences de presse et les entretiens télévisés de T. Blair (les dix plus longs, valeurs exprimées en " pour mille mots ")

\begin{tabular}{|c|c|c|c|}
\hline & Spécialisation (\%o) & Diversité (\%o) & Taille \\
\hline 19 octobre $2001^{*}$ & 15 & 380 & 3776 \\
\hline 4 avril $2002^{* *}$ & 340 & 382 & 5441 \\
\hline 11 mars $2003^{* *}$ & 200 & 383 & 4045 \\
\hline 25 mars $2003^{*}$ & 56 & 383 & 8146 \\
\hline 31 mai $2003^{* *}$ & 215 & 373 & 4165 \\
\hline 22 avril $2004^{*}$ & 54 & 363 & 8706 \\
\hline 25 mai $2004^{*}$ & 204 & 384 & 7656 \\
\hline 15 juin $2004^{*}$ & 177 & 375 & 6591 \\
\hline 28 juin $2004^{*}$ & 9 & 374 & 4065 \\
\hline 8 déc. $2004^{*}$ & 116 & 330 & 4889 \\
\hline Total conférence et entretiens $* * *$ & 163 & 398 & 162458 \\
\hline
\end{tabular}

* Conférences de presse

** Entretiens télévisés

*** 87 textes, 162458 mots.

42 La spécialisation la plus faible est observée lors de deux conférences de presse qui ont effectivement un sujet unique. Le 28 juin 2004, la conférence de presse donnée conjointement par le Premier ministre avec le chef du gouvernement provisoire irakien 
portait exclusivement sur la situation et l'avenir politique de l'Irak. Le 19 octobre 2001, T. Blair répondait aux journalistes arabes à propos des attentats du 11 septembre. A l'opposé, les trois entretiens télévisés (4 avril 2002, 11 mars 2003 et 31 mai 2003) avaient bien un sujet principal (les guerres d'Afghanistan puis d'Irak), mais d'autres sujets étaient également abordés et surtout, la manière était plus directe que dans les conférences de presse, les interviewers n'hésitant pas à interrompre T. Blair ou à le relancer.

La moyenne du tableau 2 semble refléter à peu près la propension à spécialiser le propos mais les variations autour de cette moyenne sont considérables Lorsqu'il n'y est pas contraint par ses interlocuteurs, T. Blair semble ne pas trop spécialiser ses propos. Les indices paraissent un peu plus élevés à l'oral qu'à l'écrit : le travail de rédaction consiste probablement à éliminer un certain nombre d'expressions jugées trop spécifiques par des termes plus généraux.

Enfin, les tableaux 1 et 2 permettent une comparaison éclairante entre l'écrit - il s'agit de l'écrit le plus "soutenu», puisque le discours lu par la reine doit être parfait du point de vue de la langue - avec l'oral le plus spontané (les participations de T. Blair à des émissions télévisées "populaires» ou ses discussions à bâtons rompus avec les journalistes). En français, le passage d'un de ces registres à l'autre entraînerait des différences significatives notamment en ce qui concerne la diversité du vocabulaire qui est nettement plus faible à l'oral qu'à l'écrit. Il ne semble pas en être ainsi pour T. Blair puisque la moyenne observée sur l'ensemble des entretiens est seulement inférieure à $2 \%$ par rapport à celle observée dans les discours du Trône. Nous avons d'ailleurs vu plus haut que T. Blair réalise sa meilleure performance lors d'une conférence de presse. Cependant, on remarquera que lorsque la longueur augmente, l'indice présente manifestement une tendance à la baisse. Autrement dit, les interventions orales brèves seraient plus proches de l'écrit (portant sur un nombre limité de sujets connus à l'avance, les réponses ont été mieux préparées?). On note également que, chez Tony Blair, beaucoup de passages qui sortent de l'ordinaire du point de vue de la diversité de leur vocabulaire - par le haut et, surtout par le bas - figurent dans des discours oraux. En fait, il est probable qu'une préparation écrite permet de "gommer» certains " monstres ", alors que l'oral n'autorise évidemment pas de tels remords.

Naturellement, la différence entre oral et écrit se fait probablement sentir dans d'autres dimensions du vocabulaire (par exemple, dans les densités relatives des verbes, des pronoms, des noms) ou dans les structures de phrase, etc. Des corpus comme celui qui vient d'être présenté apporteront les moyens de vérifier scientifiquement ce genre d'intuitions.

Conclusions

L'objectif essentiel n'était pas, à ce stade, d'apporter des conclusions définitives concernant le discours du Premier ministre anglais. Celui-ci étant encore en exercice à l'heure où ces lignes sont écrites, l'adjonction de nouveaux textes pourra modifier certains traits à l'avenir. Cette remarque vaut également pour les années 1997-2004 puisque le corpus devra encore être complété.

Ces limites admises, nous espérons avoir montré combien la constitution de corpus raisonnés peut être utile à la science politique et plus largement à toutes les sciences de l'homme et de la société. L'alliance de la linguistique, de la statistique et des sciences de l'informatique ouvre aux sciences humaines et sociales un vaste domaine dont le nom 
de baptême a quelque mal à se fixer: "statistique textuelle", "lexico-métrie", « textométrie » ou " logométrie » ([Mayaffre, 2004]).

Etant donné que les premiers travaux en ce domaine datent de plus de quarante ans, on peut se demander pourquoi la diffusion de ces outils est si lente parmi les chercheurs en sciences humaines et sociales. Une étude récente a dressé un tableau des principaux obstacles à laquelle se trouve confrontée la linguistique de corpus ([Fry 2004]). Dans ces obstacles, il ne faut pas négliger l'aspect fastidieux du travail préparatoire ni le fait que ces activités ne sont pas prises en compte dans la carrière des universitaires, mais cette étude souligne aussi l'importance d'un certain nombre de difficultés propres aux sciences humaines et sociales : isolement des chercheurs, faible intégration des équipes de recherche, absence de tradition d'échange des données, mauvaise circulation de l'information scientifique... Nous espérons avoir suggéré au lecteur combien il serait intéressant de surmonter au plus vite ces obstacles.

\section{BIBLIOGRAPHIE}

Abse L. (2003). Tony Blair. The Man Who Lost his Smile. Londres : Robson Books (nouvelle édition).

Beckett F. \& Hencke D. (2004). The Blairs and Their Court. Londres : Aurum Press.

Butler L. (11 décembre 2004). « How not to run a country » (entretien avec B. Johnson). The Spectator.

Fairclough N. (2000). New Labour, New Language. Londres : Routledge.

Fry J. (2004). « The Cultural Shaping of ICTs within Academic Fields: Corpus-based Linguistics as a Case Study ", Literary and Linguistic Computing 19, 3 :303-319.

Guilhaumou J. (2002). «Le corpus en analyse de discours : perspective historique », Corpus 1 :

21-49.

Habert B., Nazarenko A. \& Salem A. (1997). Les linguistiques de corpus. Paris : A. Colin.

Herdan G. (1960). Type-token Mathematics. Gravenhave : Mouton.

Hubert P., Carbonnel J.-P. \& Chaouche A. (1989). « Segmentation des séries hydrométéorologiques. Application à des séries de précipitations et de débits de l'Afrique de l'Ouest », Journal of hydrology $110: 349-367$.

Hubert P. \& Labbé D. (1988). « Un modèle de partition du vocabulaire », in D. Labbé, P. Thoiron \& D. Serant (éds) Etudes sur la richesse et la structure lexicale. Paris-Genève : Slatkine-Champion, pp. 93-114.

Hubert P. \& Labbé D. (1994). « Vocabulary Richness ». Communication au congrès de l'ALLC-ACH (Paris : La Sorbonne). Lexicometrica 0 (http://www.cavi.univ-paris3. fr/lexicometrica/).

Hockey S. \& Martin J. (1988). OCP Users' Manual. Oxford : Oxford University Computing Service. Kampfner J. (2003). Blair's Wars. Londres : Free Press. 
Knowles G. (2004). « The Notion of a 'Lemma' : Headwords, Roots and Lexical Sets », International Journal of Corpus Linguistics 9, $1: 69-81$.

Labbé C., Labbé D. \& Hubert P. (2002). « Segmentation automatique des corpus. Voyages de l'autre côté de J.-M. Le Clézio ", in A. Morin \& P. Sebillot (éds) VIe Journées Internationales d'Analyse des Données Textuelles (Saint-Malo 13-15 mars 2002). Rennes : IRISA-INRIA, Vol. 1, pp. 359-369.

Labbé C., Labbé D. \& Hubert P. (2004). « Automatic Segmentation of Texts and Corpora », Journal of Quantitative Linguistics 11, 3 : 193-213.

Mayaffre D. (2000). Le poids des mots. Le discours de gauche et de droite dans l'entre-deux-guerres. Maurice Thorez, Léon Blum, Pierre-Etienne Flandin et André Tardieu (1928-1939). Paris : Champion.

Mayaffre D. (2004). Paroles de président. Jacques Chirac (1995-2003) et le discours présidentiel sous la Ve République. Paris : Champion.

Muller C. (1977). Principes et méthodes de statistique lexicale. Paris : Hachette.

Péaquin G. (2003). Segmentation automatique des corpus. Rapport de stage. Grenoble :

Polytech'Grenoble \& Institut d'Etudes Politiques.

Prost A. (1988). « Les mots », in R. Rémond (dir). Pour une histoire politique. Paris : Seuil.

Rawnsley A. (2000). Servants of the People: The Inside Story of New Labour. Londres : Hamish Hamilton.

Richards P. (sous la direction de) (2004). Tony Blair : In His Own Words. Londres : Politico's Publishing.

Riddell P. (2004). The Unfulfilled Prime Minister : Tony Blair and the End of Optimism. Londres : Politico's Publishing.

Seldon A. (2004). Blair. Londres : Free Press.

Short C. (2004). An Honourable Deception? New Labour, Iraq, and the Misuse of Power. Londres : Free Press.

Wimmer G. \& Altmann G. (1999). « Review Article : On Vocabulary Richness », Journal of Quantitative Linguistics 6, $1: 1-9$.

\section{NOTES}

1. L'auteur remercie Cyril et Dominique Labbé qui ont mis à sa disposition les logiciels nécessaires à cette étude et qui l'ont initié à l'art de constituer et d'exploiter un corpus. Dominique Labbé a bien voulu relire une première version de ce travail.

\section{RÉSUMÉS}

Ce papier présente le corpus des discours du Premier ministre anglais (Tony Blair) prononcés de 1997 à 2004. Avant d'être intégrés dans le corpus, les textes sont corrigés, les graphies sont standardisées. On montre que ce corpus ouvre de nombreuses pistes de recherches. Deux 
exemples sont donnés: les principales ruptures thématiques et stylistiques permettent de repérer deux grandes périodes dans ces huit années ; on caractérise la richesse du vocabulaire de T. Blair à l'aide des notions de spécialisation et de diversité.

The speeches made by the British Prime Minister (Tony Blair) from 1997 to 2004

This article presents the corpus of speeches made by the British Prime Minister (Tony Blair) from 1997 to 2004. Before being included in the corpus the texts are corrected, and the spelling is standardised. It is demonstrated that this corpus opens up numerous areas of research, of which two examples are given, the main thematic and stylistic breaks that allow us to pin-point two main periods in these eight years. Finally, the richness of T. Blair's vocabulary can be measured by the use of notions of specialisation and diversity.

\section{INDEX}

Mots-clés : discours politique, Tony Blair, Grande-Bretagne, segmentation, richesse du vocabulaire, diversité du vocabulaire, spécialisation du vocabulaire, lexicométrie

\section{AUTEUR \\ EDWARD ARNOLD}

Centre for European Studies / French Department Trinity College Dublin 